\title{
Universidad, sociedad y networking: perspectivas ante el uso de las redes sociales de perfil académico profesional
}

\author{
María Purificación Subires MANCERA \\ Universidad de Málaga \\ purificacion@uma.es \\ Silvia Olmedo SAlar \\ Universidad de Málaga \\ silviaolmedo@uma.es
}

Recibido: 20/11/2012

Aceptado: 23/01/2013

\begin{abstract}
Resumen ${ }^{1}$
Actualmente, el vínculo entre Universidad y sociedad puede articularse tanto a través de los canales habituales (OTRIs, Fundaciones y Cátedras Universidad-Empresa, Spin-offs, prácticas en empresas...) como por medio de otros recursos como las redes sociales en Internet. En el ámbito de la investigación y la docencia, dichas redes se han convertido en una interesante herramienta para la construcción de comunidades de aprendizaje (Santamaría, 2008). Además, como proponemos en nuestro trabajo, pueden servir como nexo entre el mundo académico y profesional, favoreciendo la comunicación, la colaboración, el networking y la gestión y transferencia de conocimiento entre ambos. El objetivo de la presente investigación es la de conocer y valorar la predisposición de docentes, estudiantes y profesionales al uso de redes sociales de carácter académico-profesional.
\end{abstract}

Palabras clave: Redes sociales académico-profesionales, Universidad, Sociedad, networking, predisposición

\section{University, Society and Networking: Perspectives on the Use of Academic - Professional Social Networks}

\begin{abstract}
Currently, the link between university and society can be articulated through the usual channels (OTRIs, Foundations and University-Industry Chairs, Spin-offs, placements ...) and through other resources such as online social networks. In the field of research and teaching, these networks have become an interesting tool for building learning communities (Santamaria, 2008). Moreover, as we propose in our work, social networks can serve as a bridge between the academic and professional, encouraging communication, collaboration, networking and management and knowledge transfer between the two areas. The objective of this research is to know and appreciate the willingness of teachers, students and professionals to use an academic-professional social networking.
\end{abstract}

Keywords: Academic-professional social networks, University, Society, networking, predisposition

1 La presente investigación forma parte del trabajo realizado dentro del Proyecto de Innovación Educativa del Curso de Profesorado Novel de la Universidad de Málaga: "Implantación del networking y el uso de redes sociales en la enseñanza universitaria", en el que participamos las autoras de este texto, $\mathrm{M}^{\mathrm{a}}$ Purificación Subires y Silvia Olmedo, junto con los compañeros Isabel Duran, Daniel Guerrero, Isabel Ruiz y Ma Jesús Ruiz, bajo la mentoría de la profesora Bella Palomo. 


\section{Referencia normalizada}

SUBIRES MANCERA, María Purificación y OLMEDO SALAR, Silvia (2013): "Universidad, sociedad y networking: perspectivas ante el uso de las redes sociales de perfil académico profesional". Estudios sobre el mensaje periodístico. Vol. 19, núm. especial marzo, págs.: 1037-1047. Madrid, Servicio de Publicaciones de la Universidad Complutense.

Sumario: 1. Introducción. 2. Metodología. 3. Desarrollo. 4. Conclusiones. 5. Referencias bibliográficas

\section{Introducción}

\subsection{Vínculos entre Universidad y Sociedad}

La Universidad, como institución dedicada a la docencia y la investigación, cumple un papel esencial en nuestra sociedad. En ella se forma a los estudiantes para el desempeño de una profesión y se realizan investigaciones que tienen como objetivo principal contribuir al conocimiento de nuestro entorno, a la mejora de la calidad de vida de las personas y al progreso de la sociedad. Sin embargo, pese a su grado de implicación y compromiso, a veces suele achacársele cierto grado de desconexión con su entorno social y con el mundo laboral, en el cual los estudiantes deberán saber desenvolverse una vez finalicen sus estudios. Por ello resulta especialmente interesante el impulso de actuaciones que refuercen el vínculo entre ámbito académico y profesional, y sociedad en general, permitiendo que todos puedan beneficiarse mutuamente de ese intercambio.

En este sentido, son diversas las iniciativas, con orientación docente y/o investigadora, puestas en marcha por las universidades. Las OTRIS (Oficinas de Transferencia de Resultados de Investigación), por ejemplo, actúan como intermediarias entre la universidad y la empresa, en el ámbito de la investigación, dinamizando las relaciones entre ambas. Así, informan a los investigadores sobre proyectos de $\mathrm{I}+\mathrm{D}$, ofrecen ayuda técnica y colaboran en la negociación de contratos de investigación con empresas, búsqueda de financiación o gestión de patentes. (Ministerio de Educación: web).

Otro de los canales para el trasvase directo de la actividad investigadora a la sociedad son las denominadas spin-off, "empresas de nueva creación que surgen de la iniciativa de algún miembro de la comunidad universitaria partiendo de trabajos investigadores con un componente científico-tecnológico llevados a cabo en el seno de la Universidad" (OTRI Universidad de Zaragoza: web).

Las Fundaciones Universidad-Empresa, por su parte, colaboran en la transferencia de la tecnología y promoción de la innovación, desarrollan actividades de formación continua y trabajan en la inserción y desarrollo profesional de los titulados superiores (RedFue: web). Un paso más allá está en las llamadas "Cátedras Universidad-Empresa", que persiguen un vínculo más estrecho y continuado en el tiempo (Universidad de Castilla-La Mancha, 2005: web).

Las Universidades también suelen poner a disposición de sus alumnos y egresados un servicio de orientación laboral, como ayuda en su proceso de búsqueda de empleo. Y dirigidas en especial a los estudiantes universitarios se encuentran las denominadas prácticas en empresas, las cuales les permiten poner en acción, en un entorno real, los conocimientos que han ido adquiriendo durante sus estudios. 
Además, con la implantación del EEES se subraya la necesidad de trabajar directamente en la empleabilidad de los egresados. En el Documento-Marco La Integración del Sistema Universitario Español en el Espacio Europeo de Educación Superior se insiste en la importancia de la orientación profesional de los planes de estudio de Grado, señalando que los estudiantes deben desarrollar competencias básicas, transversales y específicas, "que posibiliten una orientación profesional que permita a los titulados una integración en el mercado de trabajo" (Ministerio de Educación, 2003: 7). Juan Vicente García destaca en este sentido el papel que tuvo la aprobación del Real Decreto 1393/2007, de 29 de octubre, a través del cual se refuerzan las prácticas externas (2009: 143). Así, en los nuevos planes de estudio oficiales de grado o postgrado, las prácticas en empresas o instituciones han empezado a formar parte del listado de asignaturas ofertadas, y los alumnos pueden tener una primera toma de contacto con el mundo profesional, por medio de una asignatura de su propio plan de estudios.

\subsection{Networking}

A través de las prácticas en empresas, por ejemplo, a la vez que un primer acercamiento con el mundo profesional, los estudiantes también tienen la posibilidad de practicar el networking e ir creando redes de contacto con profesionales que puedan ayudarles en su futura incorporación al mundo laboral. Esto es algo que pueden hacer desde el inicio de su vida universitaria.

El networking podría definirse como el establecimiento de redes de contacto de carácter profesional, no sólo para la búsqueda de empleo o para la búsqueda de socios para la creación de un proyecto empresarial, sino también para el mantenimiento de contacto con compañeros de trabajo, clientes... en el caso de trabajadores y directivos (López Capel, 2008: 23).

En un estudio llevado a cabo en 2001 se comprobó que "los estudiantes son conscientes de la importancia de los contactos personales en los procesos de inserción laboral, aunque sólo alrededor de un $30 \%$ procura extender su red de relaciones antes de la graduación" (Villar et al. 2001: 221). Además, se destacaba como una de las características distintivas del mercado laboral español, "el alto porcentaje de colocaciones conseguidas a través de contactos sociales" (2001: 223) lo que también es aplicable al caso de los titulados universitarios. El networking era considerado una "estrategia eficaz" en la búsqueda de empleo (2001: 224).

Actualmente, gracias a las redes sociales profesionales en Internet (LinkedIn, Xing...), esta tarea se ve enormemente facilitada. Como proponemos en nuestro proyecto, hoy día es posible el establecimiento de redes académico-profesionales, integradas dentro del propio proceso de enseñanza-aprendizaje, y que pueden contribuir tanto a la adquisición y desarrollo de competencias profesionales, como a la creación de redes de contacto profesional. Las redes sociales se convierten así en un instrumento más para el vínculo entre Universidad y Sociedad.

\subsection{Docencia y Redes sociales, encuentros necesarios}

Las redes sociales se presentan como una nueva herramienta virtual que permite el logro de un amplio abanico de posibilidades dependiendo del objetivo de su creación y diseño. 
En el campo de la educación, las peculiaridades que caracterizan la naturaleza de dichas redes incentiva con entusiasmo su integración en el escenario educativo. Entre sus potencialidades se encuentran la capacidad para promover la adquisición de habilidades digitales del alumnado y de los docentes, la posibilidad de participar de experiencias y conocimientos en comunidades virtuales, y sin duda, es un recurso que favorece de forma excepcional el networking, desde la esfera académica al ámbito profesional y viceversa. Este último propósito, entendido desde una amplia conceptualización, permitiría una combinación de encuentros entre los futuros egresados, docentes y profesionales y expertos, y sus consecuencias pueden derivar en: crear comunidades de reflexión, de debate, compartir recursos, ideas, experiencias, etc.

Las posibilidades que brindan las redes sociales en el frente educativo ha sido tenido en cuenta y el escenario actual hace gala de ello. Muchas son las experiencias educativas que se han desarrollado en las aulas, especialmente gracias a la expansión social de Facebook. Experiencias como las desarrolladas por los profesores Miguel Túñez López y José Sixto García (2011) en Facebook apuntan algunas recomendaciones sobre la red social como entorno docente que son interesantes tener en cuenta: que la red social no está concebida como plataforma docente; es un punto de encuentro colectivo y atemporal; que los contenidos se convierten en proactivos teniendo una vigencia limitada o que Facebook "es para dialogar y no para dar discursos" y además supone un trabajo extra para docentes y alumnos.

Sin embargo, aunque sea un trabajo extra para ambos, su actitud es divergente al respecto. En el ámbito universitario, se detecta un papel pasivo adoptado por los docentes frente a uno más activo de los estudiantes como evidencia el estudio realizado por Marisol Gómez, Sergio Roses y Pedro Farias (2012). A pesar de que los universitarios todavía no demuestran una tendencia entusiasta en una aplicación académica de las redes sociales, sí se evidencia en dicha investigación una actitud positiva para su utilización con fines educativos. Una explicación de este hecho, podría ser la "llamada de efecto social", como sostiene De Haro (2009), al ya que el alumnado se suma a unas relaciones sociales que van más de allá de lo meramente educativo -que sería el aprendizaje formal, y a tenor de lo requerido en la asignatura -, y en el que también se produce el aprendizaje informal.

Ante el escenario, es preciso que los docentes nos sumemos de forma activa al ritmo de evolución tecnológica que muestran los estudiantes, para evitar lo que denuncia Esteve (2009:60) cuando señala que aún se respira "el desfase entre la potencialidad de las TIC incorporadas en las aulas y la escasa renovación de los procesos pedagógicos", motivo que nos hacen perder oportunidades de promover espacios virtuales que potencien el aprendizaje autónomo y colaborativo, tal como establece el EEES.

Más allá de la aplicación de las redes sociales en los entornos docentes, debemos mencionar que dichas redes están adoptando un amplio abanico de tipologías para dar respuesta a las demandas de docentes y alumnado. En este sentido, encontramos redes sociales académicas internacionales como Academia.edu (www.academia.edu) o ResearchGate (www.researchegate.net); redes educativas nacionales enfocadas a la mejora docente como Genmatic (www.genmagic.ning.com), dirigida a las personas del 
mundo de la formación y la educación; Edutac (www.edutac.ning.com), centrada en las tecnologías y la educación; la red DIM orientada a la investigación didáctica, la innovación y multimedia; Potachovizados (www.potachovizados.grou.ps), que se presenta como una red social abierta para que todos los docentes puedan explicar qué hacen a los demás; RedBioGeo (www.redbiogeo.ning.com) para docentes de Biología y Geología o la iniciativa "Redes Sociales Educativas" (www.eduredes.ning.com), creada por el profesor Juan José de Haro, destinada a crear un espacio para el intercambio de experiencias en la administración de redes sociales educativas. Asimismo, se han creado redes en las que el alumnado también tiene el protagonismo como la RedAlumnos (redalumnos.com), que se define como una red social educativa para profesores y alumnos o Edoome (www. edoome.com), que pretende mejorar la comunicación entre las instituciones, los docentes y el alumnado.

\subsection{Proyecto de innovación educativa: propuesta piloto de red social de carácter académico-profesional}

La presente investigación se enmarca en un Proyecto de Innovación Educativa desarrollado dentro del Programa de Formación de Profesorado Novel de la Universidad de Málaga, titulado "Implantación del networking y el uso de redes sociales en la enseñanza universitaria. La experiencia de ProNetCom" (ver nota 1).

Nuestra idea de partida es la utilizar las redes sociales con una finalidad académico-profesional, de forma que puedan servir a los estudiantes tanto en el propio proceso de enseñanza-aprendizaje (a través de la orientación y el apoyo de estos profesionales y expertos en sus propias tareas académicas), como en el establecimiento de redes de contactos profesionales (networking), que puedan servirles de ayuda para su entrada en el mundo laboral.

Nuestro proyecto se compone de cinco fases de trabajo: una primera exploratoria para conocer la predisposición de los colectivos implicados a participar en una red social de estas características, una segunda de diseño, con la creación del prototipo y el diseño de acciones didácticas, una tercera de implantación, con la realización de la experiencia piloto, una cuarta de evaluación de dicha experiencia, y una quinta, de puesta en marcha de nuestra propia red social. El presente texto se enmarca dentro de la primera de las etapas, la fase exploratoria.

\section{Metodología}

Con el objeto de conocer la predisposición, tanto de estudiantes y docentes, como de profesionales y expertos a formar parte de una red social de carácter académico-profesional en el ámbito universitario, creamos tres encuestas, dirigidas respectivamente a cada uno de estos colectivos.

El uso de la encuesta se justifica teniendo en cuenta el papel que ésta tiene como "herramienta fundamental para el estudio de las relaciones sociales" (López Romo, 2000: 33) y por la posibilidad que nos ofrece para conocer la opinión de los distintos colectivos implicados.

Las encuestas, de carácter anónimo, han sido distribuidas a través de Internet, utilizando Lime Survey. En el caso de los estudiantes, los cuestionarios han sido reali- 
zados por alumnos/as de las Facultades de Ciencias de la Comunicación, Filosofía y Letras y Ciencias de la Salud (centros en los cuales imparten docencia los/as integrantes de nuestro equipo). En el de los docentes, la encuesta ha sido distribuida en la Facultad de Ciencias de la Comunicación (espacio en el que se desarrolla el plan piloto del proyecto) y entre el profesorado del Curso de Iniciación a la Innovación Educativa, perteneciente a distintas ramas de conocimiento. Respecto a los profesionales y expertos, hemos centrado nuestra atención en los pertenecientes al ámbito de la comunicación, ya que es ésta el área de conocimiento en la que se ha llevado a cabo la experiencia piloto.

Las respuestas obtenidas a través del pre-test realizado durante la primera fase exploratoria de nuestro proyecto (170 de estudiantes, 44 de docentes y 42 de profesionales y expertos) nos ha permitido obtener una primera visión acerca de la predisposición existente por parte de cada uno de estos colectivos a participar en una red social que vincule el ámbito académico y el profesional.

\section{Desarrollo}

a) Uso de redes sociales con fines personales y frecuencia de uso

Todos los colectivos encuestados afirman mayoritariamente utilizar las redes sociales en su vida personal. Así, dicen hacerlo el 86,4 por ciento de los docentes, el 98,8 de los estudiantes y 100 por 100 de los profesionales y expertos. Donde sí que se aprecian algunas diferencias es en la frecuencia de uso de dichas redes. Así, a la respuesta, "muy a menudo" sólo responden el 38,6 por ciento de los docentes, frente al 71,8 de los estudiantes, y el 78,6 de los profesionales y expertos. Se observa, por tanto, un uso más intensivo por parte de estudiantes y profesionales y expertos, y más minoritario por parte de los docentes (de los cuales un 20,4 reconocer utilizarlas poco).

b) Redes y medios sociales utilizados con fines personales

Ante la pregunta de cuáles son las redes o medios sociales que utilizan, docentes, estudiantes y profesionales coinciden en Facebook como primera opción. Así, dicen utilizarla un 71,7 por ciento de los docentes, un 86,5 por ciento de los estudiantes, y un 97,6 por ciento de los profesionales y expertos. En el resto de opciones sí que se aprecian algunas diferencias. Así, docentes y estudiantes comparten Youtube como segunda respuesta (con un 45,4 y un 82,9 por ciento, respectivamente), mientras que en el caso de los profesionales y expertos es Twitter la herramienta más utilizada después de Facebook. Los docentes emplean LinkedIn y Twitter a partes iguales, como tercera opción (con un 36,4 por ciento), seguidos de Vimeo $(15,9)$ y de Tuenti $(11,4)$, en cuarto y quinto lugar. Los estudiantes tienen Twitter como tercera opción (con un 78,2 por ciento de respuesta), Tuenti como cuarta $(75,9)$ y LinkedIn $(27,6)$, como quinta. En el caso de los profesionales y expertos, después de Facebook y Twitter aparecen Youtube $(57,1$ por ciento), LinkedIn $(54,8)$ y Tuenti $(26,2)$. Del análisis de los resultados apreciamos que, diferentes necesidades y objetivos, llevan al empleo de diferentes herramientas. 
c) Objetivos de uso de las redes sociales

Tabla 1. Elaboración propia

\begin{tabular}{|c|c|c|c|}
\hline \multicolumn{4}{|c|}{ Objetivos de uso de las redes sociales (\%) } \\
\hline Objetivos & Docentes & Estudiantes & $\begin{array}{c}\text { Profesionales } \\
\text { y expertos }\end{array}$ \\
\hline Contactar con amigos & 63,6 & 91,2 & 92,9 \\
\hline Conocer gente & 9,1 & 20 & 19,05 \\
\hline Buscar empleo & 6,8 & 26,5 & 40,5 \\
\hline $\begin{array}{c}\text { Contactar con expertos en alguna } \\
\text { materia determinada }\end{array}$ & 38,6 & 37,1 & 59,5 \\
\hline $\begin{array}{c}\text { Establecer contacto con } \\
\text { profesionales de mi mismo campo } \\
\text { de trabajo }\end{array}$ & 45,4 & 44,7 & 66,7 \\
\hline Otro & 20,4 & 14,1 & 7,1 \\
\hline
\end{tabular}

Como puede observarse en la tabla, docentes, estudiantes y profesionales y expertos coinciden plenamente en los tres primeros objetivos de uso de las redes sociales: "contactar con amigos", "establecer contacto con profesionales de mi mismo campo de trabajo" y "contactar con expertos en alguna materia determinada". Esto demuestra que, después de los amigos, es el contacto con profesionales y expertos una de las principales finalidades de empleo de estas redes para los tres perfiles encuestados.

d) Disposición a crear un nuevo perfil en una red social educativa, para participar en ella
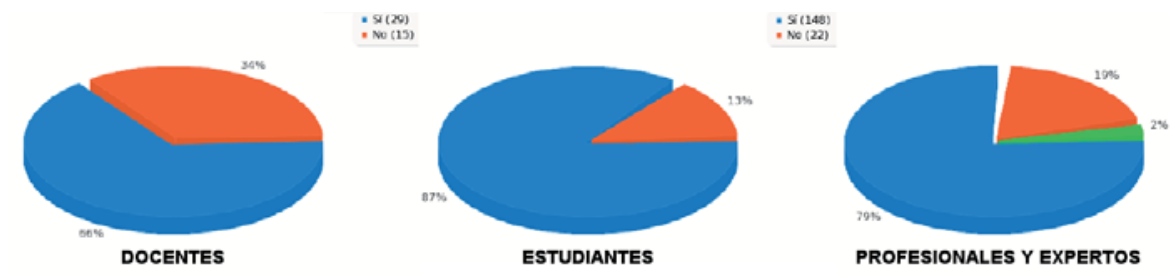

Gráfico1. Disposición a crearse un nuevo perfil en una red social educativa

La disposición a crearse un nuevo perfil en una red social educativa es mayoritaria en todos los colectivos consultados. Así, se muestran dispuestos el 65,9 por ciento de los docentes, el 87,1 por ciento de los estudiantes y el 78,6 por ciento de los profesionales y expertos. Sin embargo, observamos que el porcentaje de interés es mayor en el caso de los estudiantes que en el de los docentes y profesionales y expertos. También resulta significativo que, entre los profesionales y expertos consultados, la disposición a crearse este perfil sea mayor que entre los docentes, cuando su vinculación con el ámbito universitario no es tan directa como en el caso del profesorado.

e) Valor de una red social educativa en su desempeño profesional

Tabla 2. Elaboración propia

\begin{tabular}{|c|c|c|}
\hline \multicolumn{3}{|c|}{ Valor de una red social educativa en su desempeño profesional (\%) } \\
\hline Opciones & Docentes & $\begin{array}{c}\text { Profesionales y } \\
\text { expertos }\end{array}$ \\
\hline $\begin{array}{c}\text { Sí, sería una buena opción para contactar con } \\
\text { otras personas relacionadas con mis materias o } \\
\text { áreas de conocimiento/ con mi sector profesional }\end{array}$ & 45,4 & 33,3 \\
\hline $\begin{array}{c}\text { Sí, ayudaría al trabajo colaborativo/ o proyectos } \\
\text { entre universidad y empresa }\end{array}$ & 61,4 & 57,1 \\
\hline
\end{tabular}




\begin{tabular}{|c|c|c|}
\hline No le encuentro la utilidad & 6,8 & 0 \\
\hline No sé & 15,9 & 4,8 \\
\hline Otro & 4,5 & 4,8 \\
\hline
\end{tabular}

Respecto al valor de una red social educativa para el desempeño profesional, tanto docentes como profesionales coinciden en su utilidad para el trabajo colaborativo y la realización de proyectos entre universidad y empresa. En menor medida, y especialmente los docentes, también lo consideran una buena opción para contactar con otras personas relacionadas con su área de conocimiento o sector. Asimismo, apreciamos que es mayor, en el caso de los docentes el porcentaje de los que afirman no encontrarle utilidad o no tener muy claro que valor podría aportarles una red social en su labor docente.

En el caso de los estudiantes, las posibles aplicaciones de las redes sociales que más valoran para su empleo en clase son, por orden de importancia, en una escala del 1 al 5 (donde 5 es totalmente de acuerdo), el contacto con los compañeros (valorado con 5 por un 79,2 por ciento de ellos), la realización de trabajo colaborativo (47,6 por ciento), el contacto con los profesores $(42,9)$. Asimismo, valoran, casi un 40 por ciento con 4 y 5 , la consulta a profesionales y expertos acerca de temas concretos de la programación. En menor medida, aunque con un porcentaje superior al 30 por ciento para el valor 5, respaldan otras opciones como la realización de videoconferencias o de sesiones de chat con profesionales y expertos.

f) Disposición a participar en una red social educativa con fines académico-profesionales

Tabla 3. Elaboración propia

\begin{tabular}{|c|c|c|}
\hline \multicolumn{3}{|c|}{$\begin{array}{c}\text { Disposición a participar en una red social educativa con fines académico- } \\
\text { profesionales (desde la perspectiva académica) } \\
\text { Opciones }\end{array}$} \\
\hline $\begin{array}{c}\text { Docentes } \\
\text { Opstudiantes }\end{array}$ & Estún & 41,8 \\
\hline $\begin{array}{c}\text { Si, siempre que no significara más carga de } \\
\text { trabajo }\end{array}$ & 27,3 & 65,3 \\
\hline Si, siempre que facilitara el trabajo de clase & 47,7 & 25,9 \\
\hline $\begin{array}{c}\text { Si, siempre que me permitiera contactar con } \\
\text { expertos en temas de la asignatura }\end{array}$ & 38,6 & 28,2 \\
\hline $\begin{array}{c}\text { Si, siempre que se utilizara como medio de } \\
\text { comunicación entre compañeros entre si } \\
\text { (estudiantes-estudiantes/ docente-docente) y } \\
\text { entre docentes y estudiantes. }\end{array}$ & 36,4 & \\
\hline No sé & & 8,2 \\
\hline No & 22,7 & 1,8 \\
\hline
\end{tabular}

Tanto docentes como estudiantes coinciden en su predisposición a participar en una red social con fines académico-profesionales siempre que les facilite el trabajo en clase, aunque en el caso de los estudiantes, sí que se observa una especial preocupación en que ello no les suponga una mayor carga de trabajo, frente al caso de los profesores, que anteponen a esto el que la red pueda utilizarse como medio de comunicación entre profesores y alumnos, y como vía de contacto con expertos en el tema de la asignatura. Los estudiantes también valoran estas opciones, aunque en menor medida que los docentes. 
Tabla 4. Elaboración propia

\begin{tabular}{|c|c|}
\hline \multicolumn{2}{|c|}{$\begin{array}{c}\text { Disposición a participar en una red social educativa con fines académico- } \\
\text { profesionales (desde la perspectiva profesional) }(\%)\end{array}$} \\
\hline $\begin{array}{c}\text { Opciones } \\
\text { Profesionales y expertos }\end{array}$ \\
\hline $\begin{array}{c}\text { Si, siempre que no significara más carga de trabajo } \\
\text { Si, siempre que facilitara el trabajo habitual } \\
\text { mi área o sector }\end{array}$ \\
\hline $\begin{array}{c}\text { Si, siempre que me permitiera contactar con expertos en } \\
\text { Si, siempre que se utilizara como medio de } \\
\text { y estudiantes }\end{array}$ \\
$\begin{array}{c}\text { comunicación entre compañeros profesionales, docentes } \\
\text { profesional }\end{array}$ \\
\hline Ni, para poder vincular el mundo académico con el \\
\hline No \\
\hline
\end{tabular}

Por su parte, los profesionales y expertos valoran como principal opción a la hora de mostrar su disposición a participar en una red social de estas características, la de "poder vincular el mundo académico con el profesional", seguida de la posibilidad de que les permita contactar con expertos en su área o sector, y de que se utilice como medio de comunicación entre profesionales, docentes y estudiantes. También valoran, aunque en menor medida, el que pueda facilitarles su trabajo habitual. Sólo una cuarta parte alude a que participaría, siempre que ello no le supusiera más carga de trabajo (un porcentaje incluso menor al que registraban docentes y estudiantes). Apreciamos una buena predisposición de los profesionales y expertos encuestados a participar en una red social de estas características, siempre que permita vincular mundo académico y profesional y haga posible el contacto entre todos los colectivos implicados.

\section{Conclusiones}

Los resultados obtenidos aunque no generalizables, nos permiten observar aspectos que podemos tener en cuenta para la aplicabilidad de una red social académico-profesional:

1. Integración absoluta de las redes en la cotidianidad, el menor uso del docente: asignatura pendiente. Se constata la incursión de las redes sociales en la cotidianidad de los tres segmentos de la muestra. Sin embargo, la frecuencia de uso nos revela una interacción más limitada por parte de los docentes -casi el 50\% menor que la frecuencia de uso sostenida por profesionales y el alumnado-.

2. Las redes generalistas lideran el escenario, las demandas regulan el mapa. Las redes generalistas Facebook, Youtube y Twitter lideran las preferencias de docentes, estudiantes y profesionales, que alternan con otras generalistas y, en menor medida, con especializadas. La variedad en los usos, sugiere la idea de que las necesidades y los objetivos de los usuarios van configurando el mapa de empleo de las redes sociales.

3. El contacto personal y profesional, ejes de acción. Además de facilitar la comunicación con los amigos, las redes sociales cobran especial relevancia como mediadoras para contactar con profesionales y expertos, siendo una de las principales finalidades de su empleo, para los tres perfiles encuestados. Aspecto que sirve de guía para la aplicabilidad de una red académica-profesional.

4. Los estudiantes, agentes proactivos. Los estudiantes lideran en disposición para crear un nuevo perfil en una red social académica-profesional, con una amplia 
distancia respecto a los docentes y una menor respecto a los profesionales. Así, los estudiantes muestran un mayor empuje a la hora de hacer suyo los espacios virtuales de interacción en los que poder adquirir competencias profesionales.

5. Trabajo colaborativo, fuerza motriz. Docentes y profesionales coinciden en situar el trabajo colaborativo y la realización de proyectos entre universidad y empresa como los grandes aciertos de una red social educativa académica-profesional. Sin embargo, los estudiantes continúan situando en primer plano el contacto con los compañeros y relegan a segundo término el trabajo colaborativo, seguido del contacto con los profesores y la posibilidad de consulta con profesionales y expertos.

6. Red social académica-profesional como canal sumativo. Existen una predisposición manifiesta por parte de estudiantes, docentes y profesionales a participar en una red social académica-profesional. La suma a la iniciativa de los docentes y los estudiantes estaría motivada siempre que suponga facilitar las tareas de clase. Sin embargo, los estudiantes muestran condicionantes si supone una mayor carga de trabajo, mientras que los docentes relegan este hecho, siempre que la red facilite la comunicación entre profesores y alumnos, y permita contactar con expertos en el tema de la asignatura. Los profesionales centran su interés en la posibilidad que brinda dicha red de aunar el mundo académico con el profesional así como canal de contacto con expertos en su área o sector y vía de comunicación entre profesionales, docentes y estudiantes.

\section{Referencias bibliográficas}

DE HARO, Juan José (2009): "Las redes sociales aplicadas a la práctica docente". Revista DIM: Didáctica, Innovación y Multimedia, $\mathrm{n}^{\mathrm{o}}$ 13: http://dialnet.unirioja .es $/$ servlet/articulo? codigo $=2934817 \&$ orden $=202356 \&$ info $=$ link $[$ fecha de consulta: 01/09/2012]

ESTEVE, Francesc (2009): "Bolonia y las TIC: de la docencia 1.0 al aprendizaje 2.0". La Cuestión Universitaria, $\mathrm{n}^{\circ}$ 5, pp.59-68.

GARCÍA MANJÓN, Juan Vicente (2009): "La empleabilidad de los universitarios en el marco del Espacio Europeo de Educación Superior", en GARCÍA MANJÓN, Juan Vicente: Hacia el espacio europeo de educación superior: el reto de la adaptación de la Universidad a Bolonia, La Coruña, Netbiblo, pp.140-157.

GÓMEZ, Marisol; ROSES, Sergio; FARIAS, Pedro (2012): "El uso académico de las redes sociales en universitarios". Revista Comunicar, Vol. XIX, No 38, pp.131-138.

LÓPEZ CAPEL, Félix (2008): El poder del networking. La Coruña, Netbiblo.

MINISTERIO DE EDUCACIÓN, CULTURA Y DEPORTE (2003): "La Integración del Sistema Universitario Español en el Espacio Europeo de Enseñanza Superior. Documento-Marco", http://www.eees.es/pdf/Documento-Marco_10_Febrero.pdf [fecha de consulta: 15/10/2012]

MINISTERIO DE EDUCACIÓN, CULTURA Y DEPORTE: “Origen y funciones de las OTRI", en: http://www.educacion.gob.es/educacion/universidades/transferen- 
cia-conocimiento/oficinas-transferencia/origen-funciones-otri.html [fecha de consulta: $14 / 10 / 2012]$

OTRI UNIVERSIDAD DE ZARAGOZA: “¿Qué es Spinoff?”, http://www.unizar.es /otri/area/spin/def.php [fecha de consulta: 14/10/2012]

REDFUE: “Áreas de actividad", http://www.redfue.es/subseccion.php?id=00027 [fecha de consulta: 14/10/2012]

TÚÑEZ LÓPEZ, Miguel; SIXTO GARCÍA, José (2011): "Las redes sociales como entornos docentes: análisis del uso de Facebook en la docencia universitaria". Pixel-Bit. Revista de Medios y Educación, http://acdc.sav.us.es/pixelbit/images/stories/A11_011-V1_premaq-preprint.pdf [fecha de consulta: 02/10/2012]

VILLAR, Esperança; CAPELL, Dolors; JUAN, Jaume; y COROMINAS, Enric (2001): Educación universitaria y construcción de redes sociales como estrategia de inserción profesional. Revista de investigación educativa, RIE, Vol. 19, $\mathrm{N}^{\mathrm{o}}$ 1, pp. 221-247. http://www.doredin.mec.es/documentos/007200230131.pdf [fecha de consulta: $17 / 10 / 2012]$

\section{Ma Purificación SUBIRES MANCERA}

Universidad de Málaga

Facultad de Ciencias de la Comunicación. Departamento de Periodismo.

Licenciada en Periodismo y Máster en Desarrollos Sociales de la Cultura Artística.

Becaria de Formación de Personal Docente e Investigador (Junta de Andalucía).

purificacion@uma.es

\section{Silvia OLMEDO SALAR}

Universidad de Málaga

Facultad de Ciencias de la Comunicación. Departamento de Periodismo.

Licenciada en Periodismo y Máster en Gestión Estratégica e Innovación en Comunicación.

Becaria de Formación de Personal Docente e Investigador (Junta de Andalucía).

silviaolmedo@uma.es 